\title{
Auswirkungen von automatischen Gedanken und psychologischer Flexibilität auf die depressive Symptomatik und Rückfallwahrscheinlichkeit remittiert Depressiver
}

\author{
Roberto Rojas $^{a}$ Tabea Ig $^{a}$ Edgar Geissner ${ }^{b, c}$ Martin Hautzinger ${ }^{d}$ \\ a Institut für Psychologie und Pädagogik, Universität UIm, UIm, Deutschland; \\ b Schön Klinik Roseneck, Prien am Chiemsee, Deutschland; \\ ${ }^{c}$ Department Psychologie, Universität München, München, Deutschland; \\ ${ }^{\mathrm{d}}$ Fachbereich Psychologie, Eberhard Karls Universität Tübingen, Tübingen, Deutschland
}

\section{Schlüsselwörter}

Rezidivierende Depression - Automatische Gedanken .

Psychologische Flexibilität

\section{Zusammenfassung}

Hintergrund: Obwohl positive und negative automatische $\mathrm{Ge}$ danken (PAG, NAG) sowie die psychologische Flexibilität (PF) einen engen Zusammenhang mit der depressiven Symptomatik aufweisen, wurde die Rolle dieser Variablen bei einem depressiven Rückfall bisher nicht erforscht. Fragestellung: In dieser Studie wird untersucht, wie sich eine stationäre Intervention auf klinische Variablen (PAG, NAG und PF) auswirkt. Zudem wird der Verlauf von PAG, NAG und PF von Frührückfälligen in der Katamnesephase erforscht. Dabei geht es darum, inwiefern PAG, NAG und PF einen Rückfall vorhersagen. Methode: Bei 39 remittierten Depressiven sowie 45 gesunden Kontrollpersonen wurden die oben genannten Variablen erhoben. In der experimentellen Phase wurde bei den Probanden beider Gruppen eine traurige Stimmung induziert. Die remittiert depressiven Probanden wurden über eine 16-monatige Katamnesephase nachuntersucht. Ergebnisse: Nach der negativen Stimmungsinduktion riefen remittiert Depressive im Vergleich zu Gesunden weniger PAG ab. Zudem zeigten Frührückfällige während der Katamnesephase einen eingeschränkten Zugang zu PAG und eine niedrige PF. Weder PAG, NAG noch PF erwiesen sich als bedeutsame Prädiktoren eines depressiven Rückfalls. Schlussfolgerung: Remittiert Depressive und Frührückfällige zeigen einen eingeschränkten Zugang zu PAG. Die schwache Produktion von PAG stellt einen Risikofaktor für einen depressiven Rückfall dar.

(C) 2016 S. Karger GmbH, Freiburg

Aus Gründen der Lesbarkeit werden lediglich die männlichen Formen genannt. Gemein sind jedoch in allen Fällen sowohl Frauen als auch Männer.

\section{Keywords}

Major depression - Automatic thoughts .

Psychological flexibility

\section{Summary}

Impact of Automatic Thoughts and Psychological Flexibility on Depressive Symptoms and on Relapse of Remitted Depressed Persons

Theoretical Background: Although positive and negative automatic thoughts (PAT, NAT) and psychological flexibility (PF) are strongly related to depressive symptoms, the role of these variables in a depressive relapse has not been examined yet. Question: The study examines the impact of an inpatient treatment on clinical variables like PAT, NAT, and PF. Furthermore, the course of PAT, NAT, and PF of individuals with early relapse after therapy is observed. Finally, the study focuses on the question, to what extent PAT, NAT, and PF predict a relapse in depression. Method: The variables were measured in 39 remitted depressed subjects and 45 healthy control persons. During the experimental phase, a sad mood was induced in both groups. The remitted depressed subjects went through a 16-month follow-up phase. Results: After the negative mood induction, remitted depressed showed less PAT in comparison to healthy persons. During the follow-up phase, individuals with early relapse showed restricted access to PAT and low PF. None of the variables (PAT, NAT, PF) could predict depressive relapse. Conclusion: Remitted depressive and early relapsed subjects show limited access to PAT. The weak production of PAT represents a risk factor for a depressive relapse.

\section{KARGER}

() 2016 S. Karger GmbH, Freiburg
Dr. Roberto Rojas Institut für Psychologie und Pädagogik, Psychotherapeutische Hochschulambulanz Universität Ulm

Schaffnerstraße 3, 89073 Ulm, Deutschland roberto.rojas@uni-ulm.de 


\section{Hintergrund}

Unipolare depressive Episoden gehören zu den häufigsten und stark beeinträchtigenden psychischen Erkrankungen [Bromet et al., 2011]. In Deutschland haben depressive Störungen eine Lebenszeitprävalenz von 11-17\% und eine 12-Monats-Prävalenz von 6-12\% [Busch et al., 2013; Jacobi et al., 2004]. Im Schnitt erleiden die Betroffenen im Laufe ihres Lebens 3 bis 4 depressive Episoden [Keller und Boland, 1998]. Als Risikofaktoren für das Wiederauftreten depressiver Episoden werden eine erhöhte Anzahl vorangegangener depressiver Phasen, bestehende Restsymptomatik, kognitive Reaktivität (Definition siehe unter Abschnitt «Kognitive Reaktivität und automatische Gedanken») und chronische Belastungsfaktoren diskutiert [Burcusa und Iacono, 2007; Rojas et al., 2014; Schauenburg und Clarkin 2003; Segal et al., 2006].

\section{Automatische Gedanken und Depression}

Das «Kognitive Modell der Depression» gilt als ein einflussreicher Erklärungsansatz für depressive Rückfälle [Beck, 1967; Garrat et al., 2007; Thomsen, 2006; Winkeljohn Black und Pössel, 2015]. In diesem Modell werden 4 verschiedene Konstrukte definiert [Beck, 1967; Disner et al., 2011]: Jeder Mensch verfügt über «kognitive Schemata», die das erste Konstrukt repräsentieren. Diese verarbeiten und organisieren alle aufgenommenen Informationen und Erfahrungen. Inkludieren diese Schemata negative Sachverhalte und Überzeugungen, entstehen sogenannte «depressogene Schemata». In stressreichen Situationen werden die Schemata reaktiviert, was zu «kognitiven Verzerrungen» (zweites Konstrukt) führen kann. Als logische Schlussfolgerung der sich negativ verändernden Prozesse, entsteht die «kognitive Triade» (drittes Konstrukt). Sie besteht aus negativen Einstellungen gegenüber dem Selbst, der Welt und der Zukunft. Diese Einstellungen zeigen sich durch die «automatischen Gedanken» (AG; viertes Konstrukt) [Hollon et al., 1996; Pössel und Knopf, 2008; Winkeljohn Black und Pössel, 2015]. Bei AG handelt es sich um spontan auftretende, schnell ablaufende Gedanken, die meist selbstverständlich erscheinen. Diese können sowohl negativ als auch positiv gefärbt sein [Huffzinger et al., 2008]. Die vorliegende Arbeit beschäftigt sich im Wesentlichen mit AG als Teil des Kognitiven Modells der Depression.

Das Vorhandensein von «negativen automatischen Gedanken» (NAG) bei depressiven Patienten wurde mehrmals bestätigt [Crandell und Chambless, 1986; Dobson und Shaw, 1986; Dozois et al., 2009; Hjemdal et al., 2013; Oei und Sullivan, 1999]. Es zeigt sich, dass eine Verbesserung der depressiven Symptomatik durch Psychotherapie mit einer Reduktion der NAG [Furlong und Oei, 2002] und einer Zunahme der "positiven automatischen Gedanken» (PAG) einhergeht [Dozois, 2007; Dozois et al., 2009; Shiraishi, 2005]. Daher wird ein inverser Zusammenhang zwischen PAG und depressiver Symptomatik angenommen [Ingram et al., 1995; Missel und Sommer, 1983].

Ob davon ausgegangen werden kann, dass AG moderierend auf eine depressive Symptomatik einwirken oder eine Vulnerabilität für Depressionen durch AG vorliegt, ist umstritten [Oei und Shut- tlewood, 1996; Haaga et al., 1991]. So wird beispielsweise diskutiert, ob AG den Einfluss negativer Lebensereignisse auf die Entstehung der depressiven Symptomatik vermitteln oder ob diese den ungünstigen Effekt dysfunktionaler Einstellungen auf die depressive Symptomatik begünstigen [Kwon und Oei, 1992; Szentagotai und Freeman, 2007].

\section{Kognitive Reaktivität und automatische Gedanken}

Es gibt Belege dafür, dass eine negative bzw. traurige Stimmung zu einer Intensivierung dysfunktionaler Einstellungen bei remittiert Depressiven führen kann [Miranda und Persons, 1988; Miranda et al., 1998]. Dieses Phänomen wird als «kognitive Reaktivität» bezeichnet und ist mit einem erhöhten Risiko für einen Rückfall in die Depression assoziiert [Lethbridge und Allen, 2008; Rojas et al., 2014; Segal et al., 1999, 2006]. Dysfunktionale Einstellungen und AG sind eng verknüpft [Pössel und Knopf, 2008; Winkeljohn Black und Pössel, 2015]. Daher könnte sich bei remittiert Depressiven durch eine negative Stimmungsinduktion der Zugang zu PAG und NAG verändern. Das könnte wiederum mit einem erhöhten Risiko eines depressiven Rückfalls assoziiert sein. Diese Hypothese wurde bis jetzt noch nicht untersucht.

\section{Psychologische Flexibilität und Depression}

Eine ergänzende Sichtweise auf die Entstehung und die Aufrechterhaltung einer depressiven Symptomatik liefert das Konstrukt der «psychologischen Flexibilität» (PF), das der Akzeptanzund Commitment-Therapie (ACT) entspringt [Hayes et al., 1999; Hayes, 2004]. Personen mit einer ausgeprägten PF zeigen einen intensiven Kontakt zum gegenwärtigen Moment sowie eine höhere Akzeptanz gegenüber ihren Gefühlen und Gedanken. Dadurch werden sie befähigt, ihr Verhalten den persönlichen Werten und Situationen anzupassen, indem sie dieses flexibel ändern oder beibehalten [Hayes et al., 2006]. PF beinhaltet verschiedene Teilaspekte der ACT, wie die Entwicklung einer achtsamen Haltung und Akzeptanz, die Reduktion der Erlebensvermeidung sowie den Aufbau der kognitiven Defusion [Hoyer und Gloster, 2013]. Die kognitive Defusion wird definiert als die Lockerung der Identifikation mit sprachlichen Prozessen (vor allem Gedanken) als Folge eines achtsamen Wahrnehmens [Hayes, 2004].

PF ist ein Konstrukt, das als wesentlicher Bestandteil psychischer Gesundheit angesehen wird [Kashdan und Rottenberg, 2010]. Gemessen mit dem Fragebogen zu Akzeptanz und Handeln II (FAH-II), korreliert PF erwartungskonform negativ mit Indikatoren von Depression [Hoyer und Gloster, 2013]. Zudem wird angenommen, dass die Zunahme von PF den Einfluss der psychotherapeutischen Intervention auf die Verbesserung der depressiven Symptome vermittelt [Fledderus et al., 2013; Zettle et al., 2011].

\section{Studienziele}

Obwohl AG und PF, wie oben beschrieben, den Verlauf der depressiven Symptomatik während einer psychotherapeutischen In- 
tervention moderieren können, wurde bei remittiert Depressiven bisher noch nicht untersucht, inwiefern der Zugang zu den AG und die Ausprägung der PF einen möglichen depressiven Rückfall nach Beendigung der Behandlung beeinflussen können.

Das erste Studienziel besteht darin, die unmittelbaren Auswirkungen eines kognitiv-verhaltenstherapeutischen (stationären) Programms auf das Auftreten von PAG und NAG sowie das Niveau der PF bei depressiven Personen zu untersuchen.

Eine weitere Fragestellung bezieht sich auf die langfristige Entwicklung der Variablen (16-monatige Katamnese) und ihre Auswirkungen auf die depressive Symptomatik. Dazu wird untersucht, wie PAG, NAG und PF verlaufen und ob ein depressiver Rückfall mit Veränderungen bei diesen Variablen assoziiert ist.

Zudem gibt es bisher wenig Evidenz, ob remittiert Depressive im Vergleich zu gesunden Kontrollprobanden nach einer negativen Stimmungsinduktion (Experiment) einen veränderten Zugang $\mathrm{zu}$ PAG und NAG aufweisen. Daher wird der Frage nachgegangen, ob ein veränderter Zugang zu AG nach einer negativen Stimmungsinduktion sowie eine niedrige PF bei Entlassung die Zeit bis zu einem depressiven Rückfall während der 16-monatigen Katamnesephase vorhersagen.

\section{Methode}

\section{Design}

Die vorliegende Studie folgte einem Prä-Post-Design mit einer Katamnesephase. Am Experiment zur negativen Stimmungsinduktion nahmen sowohl remittiert depressive als auch gesunde Probanden teil. In die 16-monatige Katamnesephase wurden aber nur remittiert Depressive einbezogen. Organisatorische Gründe verhinderten die Durchführung der Katamnese bei den gesunden Probanden.

\section{Stichprobe}

An dieser Studie nahmen insgesamt 84 Personen teil; von diesen waren 39 remittiert Depressive und 45 Probanden ohne aktuelle oder vergangene psychische Störungen.

Zu Beginn des Aufenthalts an der Schön Klinik Roseneck (Prien am Chiemsee) erhielten Patienten mit einer «Major Depression» eine schriftliche Einladung zu einer Informationsveranstaltung. Bei Interesse an der Studienteilnahme unterschrieben die Patienten eine Einverständniserklärung. Zur Überprüfung der Ein- und Ausschlusskriterien für die Teilnahme an der Studie wurden das Strukturierte Klinische Interview (SKID I und II) [Wittchen et al., 1997], der Zahlen-Verbindungs-Test (ZVT) [Vernon, 1993], das Beck-Depressions-Inventar II (BDI II) [Hautzinger et al., 2006] und das Quick Inventory of Depression Symptomatology - Clinician Rating 16 Items (QIDS-C-16) [Rush et al., 2003] durchgeführt. Ausgeschlossen wurden Personen, die an schweren neurologischen oder psychischen Erkrankungen, wie dem psychotischen Syndrom, einer bipolaren Störung, Posttraumatischen Belastungsstörung, Zwangsstörung, Suchterkrankung, Essstörung und Borderline-Persönlichkeitsstörung, litten, mit Elektrokrampftherapie behandelt wurden oder die deutsche Sprache nicht ausreichend beherrschten. Der Grund dafür war, dass diese Variablen die kognitiven Prozesse sowie die korrekte Beantwortung der Fragebögen einschränken können. Im Verlauf der stationären Behandlung mit verhaltenstherapeutischem Schwerpunkt wurde die depressive Symptomatik bei allen interessierten Patienten alle 14 Tage mithilfe des BDI II erhoben. Am Experiment zur Stimmungsinduktion nahmen aber nur die Patienten teil, die mindestens 4 Wochen vor dem Experiment die Kriterien einer vollständigen Remission der depressiven Symptomatik erfüllten. Dies wurde als gegeben angenommen, wenn die Patienten im BDI II (Selbsturteil) einen Punktwert $\leq 8$ und im QIDS-C-16 (Fremdurteil) ei- nen Punktwert $\leq 5$ aufwiesen. Das Experiment zur negativen Stimmungsinduktion wurde bei den remittiert Depressiven in der Entlassungswoche durchgeführt. Genauere Angaben über die eingesetzten Fragebögen während der negativen Stimmungsinduktion finden sich im Abschnitt «Durchführung des Experiments». Von den 114 bei Klinikaufnahme zur Studie eingeladenen depressiven Patienten stimmten 62 der Teilnahme zu. 23 dieser Probanden konnten nicht an der Studie teilnehmen, weil sie mindestens 1 Ausschlusskriterium (siehe oben) erfüllten.

Die Kontrollgruppe, bestehend aus 45 gesunden Personen, wurde aus einem Probanden-Pool des Fachbereichs Psychologie der Universität Tübingen rekrutiert. Nachdem die Kontrollprobanden über das Studienziel informiert worden waren und die Einverständniserklärung unterzeichnet hatten, erfolgte die Eruierung der psychopathologischen Unauffälligkeit mithilfe des SKID I und II, des ZVT, des BDI II und des QIDS-C-16. Für die eigentliche Untersuchung wurde ein zweiter Termin vereinbart. Vor dem Experiment fand eine erneute Durchführung des BDI II statt.

Zur Sicherstellung der Vergleichbarkeit remittiert depressiver und gesunder Probanden sowie zur Kontrolle störender Variablen wurden die Gruppen bezüglich Alter, sozialer Schicht, Ausbildung und Beruf gematcht. Entsprechend fanden sich keine Unterschiede zwischen den Gruppen (alle Chi-Quadrat-Werte p > 0,10). Das Durchschnittsalter der 39 Männer und 45 Frauen lag in der Gruppe der remittiert depressiven Probanden bei 46 und in der Kontrollgruppe bei 45 Jahren. Die remittiert Depressiven litten im Schnitt seit etwa 10 Jahren an rezidivierenden Depressionen und hatten durchschnittlich 3 depressive Episoden erlebt. Bezüglich der Intensität der depressiven Symptomatik (Festlegung unter Anwendung von BDI II und QIDS-C-16) wurde bei 31 Personen (80\%) eine mittelgradige und bei $8(20 \%)$ eine schwere depressive Episode diagnostiziert. Zum Zeitpunkt der negativen Stimmungsinduktion nahmen 27 Patienten (69\%) seit mindestens 4 Wochen stabil dosiert Antidepressiva ein. Die übrigen Patienten erhielten keine Medikation. Alle Teilnehmenden galten zu diesem Zeitpunkt als remittiert.

\section{Variablen und Instrumente}

In beiden Gruppen wurden die soziodemografischen Angaben «Alter», «Geschlecht», «Familien- und Bildungsstand» und «Beschäftigungsstatus» erhoben. Außerdem wurden den Probanden Fragen zu ihrer physischen Gesundheit gestellt. Mögliche psychische Störungen der Probanden wurden durch das SKID I und II erfasst [Wittchen et al., 1997]. Im ZVT [Vernon, 1993] wurde durch die Messung von Verarbeitungsgeschwindigkeit und Konzentration das Vorliegen kognitiver Defizite überprüft.

Das BDI II [Hautzinger et al., 2006] diente dazu, in Selbstbeurteilung die Schwere der depressiven Symptomatik einzuschätzen. Das QIDS-C-16 [Rush et al., 2003] wurde eingesetzt, um die wesentlichen Symptombereiche einer Depression und deren Schwere zu beurteilen (Fremdurteil). Die 21 Items des Fragebogens positiver und negativer automatischer Gedanken (FAG) wurden unter Anwendung der Item-Korrelationswerte der Faktorenanalyse aus der Arbeit von Pössel et al. [2005] in 2 verkürzte und vergleichbare Skalen mit jeweils 11 Items unterteilt, um mögliche Effekte wiederholten Testens innerhalb eines kurzen Zeitraums zu verhindern. Die Items wurden nach der Stärke der Faktor-ItemLadung analysiert. Items jeder Subskala des FAG mit ähnlicher Faktor-ItemLadung wurden dann der entsprechenden Kurzversion zugeteilt, sodass jede Version die gleiche Anzahl von Items mit ähnlicher Ladung enthielt. Die daraus entstandenen verkürzten Versionen mit jeweils 11 Items wurden FAG-11A und FAG-11B genannt. Die Daten für die Analyse der Kurzversionen stammten aus einer Pilotstudie mit insgesamt 204 Personen (94 depressive und 110 gesunde Probanden), die vom Studienleiter durchgeführt worden war.

Die PF wurde mit der deutschen Version des Fragebogens zu Akzeptanz und Handeln II (FAH-II) [Hoyer und Gloster, 2013] gemessen. Die 20 Items des «Positive and negative Affect Schedule» (PANAS) [Krohne et al., 1996] dienen der Erfassung positiver und negativer Gefühle. Die Probanden schätzen die Intensität verschiedener Empfindungen auf einer 5-stufigen Likert-Skala von «gar nicht» (1) bis «äußerst» (5) selbst ein. Dieser Fragebogen wurde in der vorliegenden Studie vor und nach der negativen Stimmungsinduktion eingesetzt, um die Veränderung der Gefühlslage zu überprüfen. 


\section{Durchführung des Experiments}

Die experimentelle Untersuchung wurde zur Standardisierung bei allen Probanden (remittiert Depressive und Gesunde) zu einer ähnlichen Zeit (zwischen 14 und 17 Uhr) durchgeführt. Mögliche Störquellen wurden kontrolliert. Vor Beginn des Experiments füllten alle Probanden das BDI II zur Einschätzung des depressiven Status aus. Zudem wurde unter Anwendung des PANAS die aktuelle Gefühlslage erfasst. Die Probanden beider Gruppen beantworteten außerdem den FAG-11B und den FAH-II. Erst dann erfolgte die Induktion einer negativen Stimmung, indem die Probanden über Kopfhörer die 10-minütige Version der Suite Nr.1 op. 46 «Ases Tod» aus der Peer-Gynt-Suite von Edvard Grieg hörten. Währenddessen wurden die Probanden aufgefordert, an ein mindestens 2 Jahre zurückliegendes, trauriges Lebensereignis zu denken. Nach der negativen Stimmungsinduktion wurden der FAG-11A und das PANAS durchgeführt. Zum Ausgleich der Stimmungslage hörten die Probanden die 7-minütige Version der Suite Nr. 1 op. 46 «Morgenstimmung», ebenfalls aus der Peer-Gynt-Suite [siehe Rojas et al., 2014]. Dieses Vorgehen zur Stimmungsinduktion ähnelte dem anderer Arbeitsgruppen [Lethbridge und Allen, 2008; Segal et al., 1999, 2006]. Die Effektivität des Musikstücks zur negativen Stimmungsinduktion wurde in einer früheren Studie gezeigt [Rojas et al., 2014].

\section{Katamnesephase}

Nach dem Experiment begann die 16-monatige Katamnesephase. Hier wurden die remittiert Depressiven alle 4 Monate postalisch zu den AG (FAG-11A/B), zur PF (FAH-II) und zum Depressionsstatus (BDI II) befragt. Lag der BDI-IIWert über 14 (leichte depressive Symptomatik), kontaktierte der Studienleiter die Probanden innerhalb 1 Woche telefonisch, um unter Anwendung des QIDSC-16 einen möglichen depressiven Rückfall zu eruieren. Wenn auch der Wert des QIDS-C-16 $\geq 7$ lag, wurde ein depressiver Rückfall diagnostiziert. In diesem Fall erhielt der Patient im Anschluss eine telefonische Beratung.

Statistische Analysen und Datenauswertung

Mittels Chi-Quadrat-Tests wurden die Stichprobencharakteristika untersucht. Die depressive Symptomatik, die AG und die PF der remittiert Depressiven bei Aufnahme und Entlassung wurden unter Anwendung abhängiger t-Tests verglichen.

Die Ausprägung der depressiven Symptomatik bei den remittiert Depressiven und den Gesunden kurz vor der negativen Stimmungsinduktion wurde mittels univariater Varianzanalysen (analyses of variance; ANOVA) berechnet. Eine ANOVA wurde auch verwendet, um zu überprüfen, ob die negative Stimmungsinduktion für beide Gruppen gleich wirksam war. Dazu wurden die Werte auf dem PANAS vor und nach der negativen Stimmungsinduktion für beide Gruppen einbezogen. Um den Effekt der Stimmungsinduktion auf die PAG und NAG zu berechnen (Prä-Post), erfolgte der Einsatz von ANOVA mit Messwiederholung. Hier wurde das Partielle Eta-Quadrat $\left(\eta^{2}\right)$ zur Bestimmung der Effektstärke angewandt.

Die Schwankung der Eingangswerte ist ein häufiges Problem bei Studien mit einem Prä-Post-Design. Daher wurden die Indizes der automatischen Gedanken ermittelt [Brockmeyer et al., 2012; Segal et al., 2006]. Dafür wurden einfache lineare Regressionsanalysen mit den Werten vor dem Experiment (FAG-11B) als Prädiktor und den Werten nach dem Experiment (FAG-11A) als abhängige Variable berechnet. Die resultierenden «standarisierten Residuen des Modells» (Zres-FAG) stellten unverzerrte Messungen der Änderungen (Prä-Post) dieser Variablen dar [Cohen et al., 2003]. Die Indizes der PAG und NAG wurden bei den Analysen des Effekts der negativen Stimmungsinduktion verwendet. Zur Untersuchung möglicher Veränderungen der AG bei remittiert depressiven und gesunden Probanden wurden «analyses of covariance» (ANCOVA) mit dem BDI II bei Entlassung als Kovariate durchgeführt.

Die Unterschiede zwischen den rückfälligen und den nichtrückfälligen remittiert Depressiven wurden anhand von t-Tests für unabhängige Stichproben 16 Monate nach Entlassung aus der stationären Behandlung berechnet. Die Erhebungen von AG und PF nach 16 Monaten Katamnese dienten als abhängige Variablen.
Zudem wurde anhand von t-Tests untersucht, ob sich die frührückfälligen von den spät-, bzw. nichtrückfälligen remittiert Depressiven während der Katamnesephase hinsichtlich PF und AG unterschieden. Hierzu wurden innerhalb der remittiert Depressiven 2 Subgruppen gebildet. Die erste Subgruppe (Frührückfällige) bestand aus den Probanden, die bereits innerhalb der ersten 4 Monate einen depressiven Rückfall erlitten. Die zweite Gruppe (Spät- bzw. Nichtrückfällige) bestand aus den Probanden, die zu einem späteren Zeitpunkt in der 16-monatigen Katamnese einen depressiven Rückfall bzw. keinen Rückfall hatten.

Zur Untersuchung der Assoziation zwischen einem veränderten Zugang zu PAG und NAG nach der negativen Stimmungsinduktion sowie der PF vor dem Experiment mit der Auftretenswahrscheinlichkeit eines depressiven Rückfalls während der Katamnesephase wurden binäre hierarchische logistische Regressionsmodelle eingesetzt. Zur Festlegung der Effektstärke wurde f nach Cohen berechnet und angewandt.

\section{Ergebnisse}

Wie erwartet, stellte sich bei den remittiert Depressiven eine signifikante Reduktion der depressiven Symptomatik ein. Dies zeigte sich mit hohen Effektstärken durch die Werte des BDI II ( $\mathrm{t}(39)=$ $13,83 ; \mathrm{p}=0,02 ; \mathrm{d}=2,48)$ und des QIDS-C-16 ( $\mathrm{t}(39)=11,29 ; \mathrm{p}=$ $0,001 ; \mathrm{d}=2,20)$. Aber weder für die NAG $(\mathrm{t}(38)=-1,54 ; \mathrm{p}=0,13)$ noch für die PAG ( $\mathrm{t}(38)=-1,63 ; \mathrm{p}=0,11)$ konnte ein Unterschied zwischen Aufnahme- und Entlassungszeitpunkt festgestellt werden. Die PF (FAH-II) hingegen erhöhte sich zwischen diesen beiden Zeitpunkten signifikant mit mittlerer Effektstärke ( $\mathrm{t}(39)=$ 3,$60 ; \mathrm{p}=0,001 ; \mathrm{d}=0,47$ ) (Tab. 1).

Trotz einer deutlichen Verbesserung der psychischen Gesundheit wies die Patientengruppe im Vergleich zu den gesunden Probanden ein signifikant höheres Niveau der depressiven Symptomatik auf $\left(F(1,84)=8,24 ; p=0,005 ; \eta^{2}=0,10\right)$. Daher wurde der BDI-II-Wert bei Entlassung in den weiteren Analysen als Kovariat berücksichtigt.

\section{Negative Stimmungsinduktion bei remittiert Depressiven und Gesunden}

Zunächst wurde mittels einer ANOVA überprüft, ob die negative Stimmungsinduktion für beide Gruppen gleichermaßen wirksam war. Es zeigte sich ein Zeiteffekt $(\mathrm{F}(1,82)=109,31 ; \mathrm{p} \leq 0,0001$; $\left.\eta^{2}=0,57\right)$, aber kein Interaktionseffekt (Zeit $\times$ Gruppe). Die negative Stimmungsinduktion war somit für beide Gruppen gleich.

Zur Untersuchung möglicher unterschiedlicher Ausprägungen der PAG und NAG vor und nach der Stimmungsinduktion wurden Indizes der AG berechnet und zur Analyse eingesetzt. Unter Anwendung einer ANCOVA mit BDI II zur Entlassung, deren Werte als Kovariate verwendet wurden, konnte eine deutliche $\mathrm{Zu}$ nahme der PAG bei den gesunden Kontrollpersonen ( $M=0,44 \pm$ 0,81 ) und eine deutliche Abnahme dieses Werts bei den remittiert Depressiven $(\mathrm{M}=-0,51 \pm 0,95)$ mit großem Effekt festgestellt wer$\operatorname{den}\left(F(2,84)=12,77 ; p=0,001 ; \eta^{2}=0,24\right)$. Hinsichtlich der NAG zeichnete sich kein Unterschied zwischen beiden Gruppen ab (remittiert Depressive: $\mathrm{M}=0,09 \pm 1,1$; Kontrollgruppe: $\mathrm{M}=-0,08 \pm$ $0,88)(\mathrm{F}(2,82)=0,31 ; \mathrm{p}=0,73)$. 
Tab. 1. Deskriptive Statistik der demografischen und der Studienvariablen von remittiert depressiven und gesunden Probanden bei Aufnahme sowie vor und nach der negativen Stimmungsinduktion

\begin{tabular}{|c|c|c|}
\hline Studienvariablen & $\begin{array}{l}\text { Remittiert } \\
\text { Depressive } \\
(\mathrm{n}=39)\end{array}$ & $\begin{array}{l}\text { Gesunde } \\
(\mathrm{n}=45)\end{array}$ \\
\hline \multicolumn{3}{|l|}{ Demografische Daten } \\
\hline Alter, Jahre $(\mathrm{M} \pm \mathrm{SD})$ & $46 \pm 9$ & $5 \pm 9$ \\
\hline Geschlecht, $\mathrm{n}(\mathrm{m} / \mathrm{w})$ & $18 / 21$ & $21 / 24$ \\
\hline Abitur, n (\%) & $21(54)$ & $26(58)$ \\
\hline Berufstätigkeit, n (\%) & $33(85)$ & $39(87)$ \\
\hline \multicolumn{3}{|l|}{ Depressive Symptomatik } \\
\hline BDI II bei Aufnahme $(\mathrm{M} \pm \mathrm{SD})$ & $23 \pm 5$ & - \\
\hline BDI II vor Experiment $(\mathrm{M} \pm \mathrm{SD})$ & $7 \pm 2$ & $5 \pm 2$ \\
\hline QIDS-C-16 bei Aufnahme $(\mathrm{M} \pm \mathrm{SD})$ & $2,64 \pm 1,31$ & - \\
\hline QIDS-C-16 vor Experiment $(\mathrm{M} \pm \mathrm{SD})$ & $1,30 \pm 0,50$ & $1,0 \pm 0,20$ \\
\hline \multicolumn{3}{|l|}{$\mathrm{PF}$} \\
\hline FAH-II bei Aufnahme $(\mathrm{M} \pm \mathrm{SD})$ & $30 \pm 7,40$ & - \\
\hline FAH-II vor Experiment $(\mathrm{M} \pm \mathrm{SD})$ & $34 \pm 9,50$ & $44 \pm 5,80$ \\
\hline \multicolumn{3}{|l|}{ NAG } \\
\hline FAG-11A Aufnahme $(\mathrm{M} \pm \mathrm{SD})$ & $1,90 \pm 0,80$ & - \\
\hline FAG-11B vor Experiment $(\mathrm{M} \pm \mathrm{SD})$ & $2,10 \pm 0,70$ & $1,40 \pm 0,40$ \\
\hline FAG-11A nach Experiment $(\mathrm{M} \pm \mathrm{SD})$ & $1,80 \pm 0,80$ & $1,30 \pm 0,50$ \\
\hline Index der NAG $(\mathrm{M} \pm \mathrm{SD})$ & $0,09 \pm 1,10$ & $-0,08 \pm 0,90$ \\
\hline \multicolumn{3}{|l|}{ PAG } \\
\hline FAG-11A Aufnahme $(\mathrm{M} \pm \mathrm{SD})$ & $3,60 \pm 0,90$ & - \\
\hline FAG-11B vor Experiment $(\mathrm{M} \pm \mathrm{SD})$ & $3,34 \pm 0,85$ & $3,20 \pm 0,84$ \\
\hline FAG-11A nach Experiment $(\mathrm{M} \pm \mathrm{SD})$ & $3,94 \pm 1,11$ & $2,80 \pm 0,95$ \\
\hline Index der PAG $(\mathrm{M} \pm \mathrm{SD})$ & $-0,51 \pm 0,90$ & $0,44 \pm 0,80$ \\
\hline \multicolumn{3}{|l|}{ Emotionaler Zustand } \\
\hline PANAS - neg. Affekt vor Experiment $(\mathrm{M} \pm \mathrm{SD})$ & $1,30 \pm 0,30$ & $1,30 \pm 0,30$ \\
\hline PANAS - neg. Affekt nach Experiment $(\mathrm{M} \pm \mathrm{SD})$ & $1,80 \pm 0,60$ & $1,90 \pm 0,40$ \\
\hline \multicolumn{3}{|c|}{$\begin{array}{l}\text { M = Mittelwert; SD = Standardabweichung; PF = psychologische Flexibilität; NAG = negative automa- } \\
\text { tische Gedanken; PAG = positive automatische Gedanken; neg. = negativ; BDI II = Beck-Depressions- } \\
\text { Inventar II; QIDS-C-16 = Quick Inventory of Depression Symptomatology - Clinician Rating } 16 \text { Items; } \\
\text { FAG-11A/B = Fragebogen positiver und negativer automatischer Gedanken (11A und 11B); FAH-II = } \\
\text { Fragebogen zu Akzeptanz und Handlung II; PANAS = Positive and Negative Affect Schedule. }\end{array}$} \\
\hline
\end{tabular}

\section{Langfristige Effekte}

Vergleich zwischen rückfälligen und nichtrückfälligen remittiert Depressiven nach 16 Monaten Katamnese

Im Laufe der Katamnesephase erlitten 18 Probanden (46\%) einen depressiven Rückfall. Davon waren 12 weiblich und 6 männlich. Unter Anwendung von t-Tests für unabhängige Stichproben wurde untersucht, ob Unterschiede bezüglich AG und PF zwischen allen Rückfälligen und Nichtrückfälligen nach 16 Monaten Katamnese bestanden. Zu diesem Messzeitpunkt zeigten die remittiert Depressiven ohne einen Rückfall ( $n=21)$ im Vergleich zu den Rückfälligen sowohl eine höhere PF als auch einen stärkeren Zugang zu PAG. Diese Ergebnisse sind signifikant mit großen Effektstärken. Im Gegensatz dazu wiesen die Rückfälligen einen signifikant höheren Zugang zu NAG als die Nichtrückfälligen auf (Tab. 2).

Vergleich zwischen Frührückfälligen und Spät- bzw. Nichtrückfälligen remittiert Depressiven

Für ein besseres Verständnis des Einflusses der Studienvariablen auf einen frühen depressiven Rückfall wurden innerhalb der Gruppe der remittiert Depressiven 2 Subgruppen gebildet (Gruppe
1: Frührückfällige, die innerhalb von 4 Monaten einen Rückfall erlitten; Gruppe 2: Spät- bzw. Nichtrückfällige). Innerhalb der ersten 4 Monate war die höchste Rückfallinzidenz festzustellen (Frührückfällige; $\mathrm{n}=12$ ). In den nächsten 12 Monaten erlitten weitere 6 Personen einen Rückfall (Spätrückfällige).

Bezüglich der PAG (FAG-11A/B) unterschieden sich beide Gruppen signifikant mit einer großen Effektstärke ( $\mathrm{t}(37)=2,25 ; \mathrm{p}=$ 0,$03 ; d=0,78$ ), wobei die Frührückfälligen deutlich geringere PAG $(\mathrm{M}=1,7 \pm 0,5)$ aufwiesen als die Spät- bzw. Nichtrückfälligen $(\mathrm{M}=$ $2,7 \pm 0,6)$. Für die NAG war auch hier kein Unterschied zwischen beiden Gruppen festzustellen. Für die PF (FAH-II) zeigten die Frührückfälligen $(M=27,6 \pm 7)$ im Vergleich zu Spät- bzw. Nichtrückfälligen $(M=36,6 \pm 9,3)$ ebenfalls signifikant niedrigere Werte $(\mathrm{t}(37)=$ $3,0 ; p=0,005 ; d=1,04)$. Da eine hohe Anzahl an vorangegangenen depressiven Episoden als Risikofaktor für einen depressiven Rückfall gilt, wurde anschließend der Zusammenhang dieser Variablen mit einem frühen Rückfall untersucht. Beide Gruppen (Frührückfällige: $M=4,2 \pm 2,6$; Spät- bzw. Nichtrückfällige: $M=2,0 \pm 1,1)$ unterschieden sich erwartungskonform signifikant bezüglich der Anzahl depressiver Episoden in der Vorgeschichte $(\mathrm{t}(36)=-3,71 ; \mathrm{p}=0,001$; $\mathrm{d}=1,30 ; 95 \%$-Konfidenzintervall $=-2,03,-0,55)$. 
Tab. 2. Ergebnisse der t-Tests zum Vergleich von rückfälligen und nichtrückfälligen remittiert depressiven Probanden nach 16 Monaten Katamnese bezüglich der Studienvariablen, die während der Katamnese erhoben wurden

Tab. 3. Logistische Regressionsanalyse des depressiven Rückfalls als Funktion demografischer, klinischer und kognitiver Variablen

\begin{tabular}{|c|c|c|c|c|c|}
\hline Variable & Depressiver Rückfall & $\mathrm{n}$ & $\mathrm{M} \pm \mathrm{SD}$ & $95 \% \mathrm{CI}$ & $\mathrm{d}$ \\
\hline \multirow[t]{2}{*}{ NAG (FAG-11) } & ja & 17 & $2,91 \pm 0,93$ & $-1,91,-0,65$ & $-1,26^{\star *}$ \\
\hline & nein & 21 & $1,94 \pm 0,61$ & & \\
\hline \multirow[t]{2}{*}{ PAG (FAG-11) } & ja & 17 & $1,98 \pm 0,81$ & $0,33,1,68$ & $1,05^{\star}$ \\
\hline & nein & 21 & $2,84 \pm 0,89$ & & \\
\hline \multirow[t]{2}{*}{ PF (FAH-II) } & ja & 17 & $26,60 \pm 10,00$ & $0,56,1,96$ & $1,48^{* *}$ \\
\hline & nein & 21 & $37,50 \pm 7,38$ & & \\
\hline
\end{tabular}

Bei den Rückfälligen fehlen die Daten eines Teilnehmers, daher $\mathrm{n}=17 . \mathrm{M}=$ Mittelwert; $\mathrm{SD}=$ Standardabweichung; $\mathrm{PF}=$ psychologische Flexibilität, NAG = negative automatische Gedanken; PAG = positive automatische Gedanken; 95\% CI = 95\%-Konfidenzintervall; $\mathrm{d}=$ Effektstärke nach Cohen mit Angabe des Signifikanzniveaus $\left({ }^{*} \mathrm{p}<0,01 ;{ }^{* *} \mathrm{p}<0,001\right)$; FAG-11A/B = Fragebogen positiver und negativer automatischer Gedanken (11A und 11B); FAH-II = Fragebogen zu Akzeptanz und Handlung II.

\begin{tabular}{|c|c|c|c|c|c|c|c|}
\hline Variable & Block & $\mathrm{B}$ & Wald & $e^{\beta}$ & $\chi^{2}$ (block) & $\mathrm{R}^{2}$ & f \\
\hline \multicolumn{5}{|l|}{ Analyse 1 (gemeinsame Eingabe) } & \multirow[t]{4}{*}{$5,63^{\star}$} & \multirow[t]{4}{*}{0,18} & \multirow[t]{4}{*}{0,47} \\
\hline Geschlecht & 1 & - & n.s. & - & & & \\
\hline Anzahl depressiver Episoden & 1 & 0,44 & $4,53^{*}$ & 1,55 & & & \\
\hline BDI II bei Entlassung & 1 & - & n.s. & - & & & \\
\hline \multicolumn{8}{|l|}{ Analyse 2 (schrittweise Eingabe) } \\
\hline \multicolumn{7}{|l|}{ automatischer Gedanken (FAG-11A/B) } & - \\
\hline \multicolumn{8}{|l|}{ Analyse 3 (schrittweise Eingabe) } \\
\hline $\begin{array}{l}\text { Psychologische Flexibilität vor } \\
\text { Experiment (FAH-II) }\end{array}$ & 2 & - & n.s. & - & - & - & - \\
\hline \multicolumn{8}{|c|}{$\begin{array}{l}{ }^{*} \mathrm{p}<0,05 ; \mathrm{n} . \mathrm{s} .=\text { nicht signifikant. } \\
\beta=\text { Beta; Wald = Wald-Kriterium; } \mathrm{e}^{\beta}=\text { Exponierte Beta; } \chi^{2}=\text { Chi Quadrat; } \mathrm{R}^{2}=\text { Nagelkerk'scher Korrelationswert; } \mathrm{f}=\text { Effektstärke } \\
\text { nach Cohen; BDI II = Beck-Depressions-Inventar II; FAG-11A/B = Fragebogen positiver und negativer automatischer Gedanken } \\
(11 \mathrm{~A} \text { und 11B); FAH-II = Fragebogen zu Akzeptanz und Handlung II. }\end{array}$} \\
\hline
\end{tabular}

Vorhersage eines depressiven Rückfalls bei remittiert depressiven

\section{Probanden}

Wir postulierten, dass remittiert depressive Probanden mit einem veränderten Zugang zu NAG und PAG durch die negative Stimmungsinduktion und mit einer niedrigen PF vor dem Experiment einen depressiven Rückfall erleiden würden. Zur Untersuchung der Auftretenswahrscheinlichkeit eines depressiven Rückfalls wurden binäre logistische Regressionsanalysen eingesetzt.

In einem ersten Schritt erfolgte die Überprüfung des Einflusses demografischer und klinischer Variablen (Geschlecht, Anzahl depressiver Episoden und BDI II bei Entlassung) auf die Rückfallwahrscheinlichkeit nach 16 Monaten. Das Gesamtmodell ist signifikant und klärt 18,4\% der Varianz auf $\left(\chi^{2}(4, \mathrm{~N}=38)=5,63 ; \mathrm{p}=\right.$ 0,$\left.02 ; \mathrm{R}^{2}=0,18 ; \mathrm{f}=0,47\right)$. Unter Berücksichtigung des Wald-Kriteriums kommt allerdings nur der Variable «Anzahl depressiver Episoden» Vorhersagekraft $\mathrm{zu}\left(\chi^{2}(1, \mathrm{~N}=38)=4,53 ; \mathrm{p}=0,033 ; \mathrm{e}^{\beta}=1,55\right)$.

In weiteren Einzelanalysen wurden zusätzlich die Indizes der PAG und NAG (bezogen auf FAG-11A/B) sowie die PF vor dem Experiment (bezogen auf FAH-II) berücksichtigt. Durch die Einführung dieser Variablen ins Regressionsmodell konnte jedoch keine Erhöhung der Vorhersagekraft erzielt werden, sodass die Anzahl der depressiven Episoden als einziger signifikanter Prädiktor im Modell verblieb (Tab. 3).

\section{Diskussion}

Unsere Ergebnisse zeigen eine deutliche Verbesserung der PF bei depressiven Personen, die nach einer stationären verhaltenstherapeutischen Maßnahme von ihrer depressiven Symptomatik remittiert waren. Entgegen unserer Erwartung blieben in dieser Gruppe aber signifikante Veränderungen der PAG und NAG aus. Zur Erklärung dieses Ergebnisses kann die Zustandseigenschaft (state) von AG [Beck et al., 1994; Kelava und Schermelleh-Engel, 2012] im Vergleich zur festen Persönlichkeitseigenschaft (trait) der dysfunktionalen Einstellungen [Beck et al., 1994; Rojas et al., 2014; Segal et al., 1999, 2006] herangezogen werden. So spiegelt die Ausprägung der AG vor allem den Ist-Zustand bei Entlassung wider. Zu diesem Zeitpunkt ist die emotionale Lage von remittiert depressiven Probanden als ambivalent zu bezeichnen, da einerseits Freude und Optimismus angesichts der baldigen Rückkehr ins heimische Umfeld vorhanden sind, andererseits auch Unsicherheit in Bezug auf neue Aufgaben und Entscheidungen. Wie früher im Rahmen des kognitiven Modells depressiver Störungen erwähnt, haben AG eine enge Verbindung zu dysfunktionalen Einstellungen [Pössel und Knopf, 2008; Winkeljohn Black und Pössel, 2015]. Es ist anzunehmen, dass bei der Wiederaktivierung dysfunktionaler Einstellungen (trait), ausgelöst durch Stress bzw. Belastung, auch der Zugang zu den AG (state) verändert wird. 
Bei den remittiert Depressiven führten wir eine negative Stimmungsinduktion durch und verglichen diese Patienten mit gesunden Probanden. Entgegen der Erwartung zeigte sich kein Unterschied hinsichtlich der NAG. Bei den PAG hingegen war ein signifikanter Unterschied zugunsten der gesunden Probanden zu beobachten. Dies deutet darauf hin, dass durch das Erleben einer traurigen Stimmung bei allen Probanden eine ähnliche Aktivierung der NAG auftrat. Die gesunden Probanden zeigten aber eine deutliche Zunahme von PAG angesichts der traurigen Stimmung, was als eine Bewältigungsstrategie interpretiert werden kann. Remittiert Depressive haben dagegen Schwierigkeiten, beim Erleben einer traurigen Stimmung positive und stimmungsaufhellende Gedanken abzurufen. Brockmeyer et al. [2012] untersuchten denselben $\mathrm{Zu}$ sammenhang in Bezug auf die Verwendung positiv und negativ gefärbter Emotionswörter nach einer negativen Stimmungsinduktion. Sie beobachteten, dass remittiert Depressive weniger positiv getönte Emotionswörter verwendeten als gesunde Kontrollprobanden.

Ein weiteres Ziel dieser Arbeit bestand darin, den Verlauf der klinischen Variablen bei remittiert Depressiven während der Katamnese zu untersuchen. 16 Monate nach Interventionsende konnten bei den Nichtrückfälligen im Vergleich zu den Rückfälligen ein stärkerer Zugang zu PAG sowie eine geringeres Ausmaß an NAG beobachtet werden. Unsere Befunde bestätigen somit die Ergebnisse früherer Studien [Dozois, 2007; Dozois et al., 2009; Furlong und Oei, 2002; Shiraishi, 2005]. Die Nichtrückfälligen wiesen außerdem eine deutlich höhere PF auf. Das spricht für eine schützende Funktion der PF gegen einen depressiven Rückfall.

Beim Vergleich der Frührückfälligen mit den Spät- bzw. Nichtrückfälligen zeigte sich, dass Frührückfällige von häufigeren depressiven Episoden in der Vorgeschichte berichteten. Zudem wiesen sie weniger Zugang zu PAG und eine niedrige PF auf. Hier fanden wir jedoch keinen Unterschied zwischen den Gruppen hinsichtlich der NAG. Dieses Ergebnis steht im Einklang mit unserem früheren Befund, wonach der Zugang zu PAG bei remittiert Depressiven im Vergleich zu Gesunden eingeschränkt ist.

Die Befunde lassen vermuten, dass eher der reduzierte Zugang zu den PAG als die Zunahme von NAG mit einer depressiven Symptomatik (Restsymptomatik zum Ende des Aufenthalts bzw. depressiver Rückfall in der Katamnese) assoziiert ist. Eine mögliche Interpretation dieser Ergebnisse ist, dass mit zunehmender Anzahl depressiver Episoden eine sukzessive Reduktion des Zugangs zu hilfreichen Kognitionen erfolgt bzw. sich mit jeder weiteren depressiven Episode die dysfunktionalen Einstellungen intensivieren [Lewinsohn et al., 1999; Nolen-Hoeksema et al., 1992]. Auf der anderen Seite fungieren PAG (z.B. optimistische Gedanken über das Selbst) als Puffer gegenüber drohenden Erschütterungen des Selbstwerts in belastenden Situationen [vgl. Bosson et al., 2003]. Als Implikation für die verhaltenstherapeutische Praxis ergibt sich, dass gemeinsam mit den Patienten insbesondere am Aufbau hilfreicher und vor allem realistischer Gedanken gearbeitet werden sollte. Die klinische Erfahrung zeigt, dass sich wenige Patienten darüber bewusst sind, in welchen Mustern sie denken. Daher sollten die Betroffenen lernen, unter Anwendung meta-kognitiver und/oder achtsamkeitsbasierter Methoden eine bewusste und auf- merksame Haltung gegenüber ihren Denkinhalten und Denkprozessen aufzubauen. Darauf basierend spielt die Entwicklung von Strategien zum Umgang mit NAG und dysfunktionalen Kognitionen sowie zum Aufbau stimmungsfördernder Aktivitäten und Gedanken eine wichtige Rolle. Das Praktizieren dieser Strategien, z.B. der Abruf von PAG in belastenden oder stressreichen Situationen, sollte im Alltag durch Verhaltensexperimente eingeübt werden. Auffällig ist, dass die PF bei Nichtrückfälligen höher ist als bei Rückfälligen. Daher empfiehlt es sich, die PF durch den Einsatz von ACT-Verfahren zu fördern [Hayes, 2004].

Wir haben zudem untersucht, ob sich der veränderte Zugang zu AG nach einer negativen Stimmungsinduktion und die PF bei Entlassung als Prädiktoren eines depressiven Rückfalls während der 16-monatigen Katamnesephase erweisen. Entgegen unseren Erwartungen fanden wir keine Evidenz für diese Hypothese. Als bedeutsamer Prädiktor stellte sich nur die Anzahl depressiver Episoden in der Vorgeschichte heraus. Dieses Ergebnis könnte auf die Zustandseigenschaft (state) der AG zurückgeführt werden und ergänzt die Ergebnisse früherer Studien. In diesen erwies sich die Aktivierung irrationaler Einstellungen (trait) nach einer negativen Stimmungsinduktion als bedeutsamer Prädiktor eines frühen depressiven Rückfalls [Rojas et al., 2014; Scher et al., 2005; Segal et al., 1999, 2006].

Bei der Interpretation der Ergebnisse sind jedoch einige Einschränkungen zu berücksichtigen. Wir konnten unter den naturalistisch-klinischen Rahmenbedingungen kein randomisiertes Studiendesign wählen. Auch die relativ kurze Zeitspanne zwischen der Remission der depressiven Symptomatik und der Durchführung des Experiments (4 Wochen) könnte als Einschränkung gesehen werden. Die Stichprobe, vor allem die der remittiert depressiven Probanden, war zudem relativ klein. Daher sind die Ergebnisse der Katamnesephase zu den Frührückfälligen und den Spät- bzw. Nichtrückfälligen zum jetzigen Zeitpunkt eher als explorativ und hypothesengenerierend zu bewerten.

Zur Absicherung der Befunde empfehlen wir, ehemalige Depressive mit kurzer Remissionszeit und Probanden, die als von der depressiven Phase genesen gelten, zu vergleichen. Für die klinische Praxis wäre es relevant zu untersuchen, ob sich eine klassische Kognitive Verhaltenstherapie der Depression durch Zusatzmodule zur Förderung der PF und PAG, beispielsweise durch meta-kognitive Strategien, verbessern lässt. Zusammenhänge zwischen dysfunktionalen Einstellungen und AG sollten im Rahmen von experimentellen Ansätzen validiert werden. Dies sollte zur weiteren Erhellung der Binnen- und Zusammenhangsstruktur der hier vorgestellten Konstrukte beitragen und könnte wichtige Implikationen für die Diagnose und Behandlung depressiver Störungen haben.

\section{Ethikvotum}

Das Studienprotokoll mitsamt den Aufklärungsinformationen und Einverständniserklärungen wurde von der Ethik-Kommission des Universitätsklinikums Tübingen geprüft und genehmigt. Alle Teilnehmer erhielten mündliche und schriftliche Informationen über die Studie. Eine schriftliche Einverständniserklärung wurde zum Studienbeginn unterschrieben. 


\section{Disclosure Statement}

Die Autoren erklären hiermit, dass keinerlei Interessenskonflikte in Bezug auf das vorliegende Manuskript bestehen.

\section{Literatur}

Beck AT: Depression: Clinical, Experimental, and Theoretical Aspects. New York, Harper \& Row, 1967.

Beck AT, Rush J, Shaw BF, Emery G: Kognitive Therapie der Depression. Weinheim, Psychologie Verlags Union, 1994.

Bosson JK, Brown GK, Zeigler-Hill V, Swann WB: Selfenhancement tendencies among people with high explicit self-esteem: the moderating role of implicit selfesteem. Self Identity 2003;2:169-187.

Brockmeyer T, Pfeiffer N, Grosse-Holforth M, et al: Mood regulation and cognitive reactivity in depression vulnerability. Cognit Ther Res 2012;36:634-642.

Bromet E, Andrade LH, Hwang I, et al: Cross-national epidemiology of DSM-IV major depressive episode. BMC Med 2011;9:90.

Burcusa SL, Iacono WG: Risk for recurrence in depression. Clin Psychol Rev 2007;27:959-985.

Busch MA, Maske UE, Ryl L, et al: Prävalenz von depressiver Symptomatik und diagnostizierter Depression bei Erwachsenen in Deutschland. Bundesgesundheitsblatt 2013;56:733-739.

Cohen J, Cohen P, West SG, Aiken LS: Applied Multiple Regression/Correlation Analysis for the Behavioral Sciences, ed 3rd. New Jersey, Lawrence Erlbaum Associates, 2003

Crandell CJ, Chambless DL: The validation of an inventory for measuring depressive thoughts: the Crandell Cognitions Inventory. Behav Res Ther 1986;24:403-411.

Disner SG, Beevers CG, Haigh EA, Beck AT: Neural mechanisms of the cognitive model of depression. Nat Rev Neurosci 2011;12:467-477.

Dobson KS, Shaw BF: Cognitive Assessment with major depressive disorders. Cognit Ther Res 1986;10:13-29.

Dozois DJ: Stability of negative self-structures: a longitudinal comparison of depressed, remitted, and nonpsychiatric controls. J Clin Psychol 2007;63:319-338.

Dozois DJ, Bieling PJ, Patelis-Siotis I, et al: Changes in selfschema structure in cognitive therapy for major depressive disorder: a randomized clinical trial. J Consult Clin Psychol 2009;77:1078-1088.

Fledderus M, Bohlmeijer ET, Fox JP, et al: The role of psychological flexibility in a self-help acceptance and commitment therapy intervention for psychological distress in a randomized controlled trial. Behav Res Ther 2013;51:142-151.

Furlong M, Oei TP: Changes to automatic thoughts and dysfunctional attitudes in-group CBT for depression. Behav Cogn Psychother 2002;30:351-360.

Garratt G, Ingram RE, Rand KL, Sawalani G: Cognitive processes in cognitive therapy: evaluation of the mechanisms of change in the treatment of depression. Clin Psychol (New York) 2007;14:224-239.

Haaga DA, Dyck MJ, Ernst D: Empirical status of cognitive therapy of depression. Psychol Bull 1991;110:215-236.

Hautzinger M, Keller F, Kühner C: Beck Depressions-Inventar II (BDI II), Testhandbuch. Frankfurt/M., Harcourt Test Services, 2006.

Hayes SC, Luoma J, Bond F, et al: Acceptance and commitment therapy: model, processes, and outcomes. Behav Res Ther 2006;44:1-25.

Hayes SC, Strosahl KD, Wilson KG: Acceptance and Commitment Therapy: an Experiential Approach to Behavior Change. New York, Guilford Press, 1999.
Hayes SC: Acceptance and commitment therapy, relational frame theory, and the third wave of behavioral and cognitive therapies. Behav Ther 2004;35:639-665.

Hjemdal O, Stiles T, Wells A: Automatic thoughts and meta-cognition as predictors of depressive or anxious symptoms: a prospective study of two trajectories. Scand J Psychol 2013;54:59-65.

Hollon SD, DeRubeis RJ, Evans MD: Cognitive therapy in the treatment and prevention of depression; in Salkovskis PM (ed): Frontiers of Cognitive Therapy. New York, Guilford, 1996, pp 293-317.

Hoyer J, Gloster AT: Psychologische Flexibilität messen: Der Fragebogen zu Akzeptanz und Handeln II (FAHII). Verhaltenstherapie 2013;23:42-44.

Huffzinger S, Meyer TD, Seemann S, et al: Evaluation eines deutschsprachigen Instrumentes zur Erfassung positiver und negativer automatischer Gedanken bei Kindern und Jugendlichen. Z Klin Psychol Psychother 2008;37:255-262.

Ingram RE, Kendall PC, Siegle G, et al: Psychometric properties of the positive automatic thoughts questionnaire. Psychol Assessment 1995;7:495-507.

Jacobi F, Wittchen HU, Hölting C, et al: Prevalence, comorbidity and correlates of mental disorders in the general population: results from the German Health Interview and Examination Survey (GHS). Psychol Med 2004;34:1-15.

Kashdan TB, Rottenberg J: Psychological flexibility as a fundamental aspect of health. Clin Psychol Rev 2010; 30:865-878.

Kelava A, Schermelleh-Engel K: Latent-State-Trait-Theorie (LST Theorie); in Moosbrugger H, Kelava A (eds): Testtheorie und Fragebogenkonstruktion. Berlin, Springer, 2012, pp 362-380.

Keller MB, Boland RJ: Implications of failing to achieve successful long-term maintenance treatment of recurrent unipolar major depression. Biol Psychiatry 1998; 44:348-360.

Krohne HW, Egloff B, Kohlmann CW, Tausch A: Untersuchungen mit einer deutschen Version der «Positive und Negative Affekt Schedule» (PANAS). Diagnostica 1996;42:139-156.

Kwon SM, Oei TPS: Differential causal roles of dysfunctional attitudes and automatic thoughts in depression. Cognit Ther Res 1992;16:309-328.

Lethbridge R, Allen N: Mood induced cognitive und emotional reactivity, life stress, and the prediction of depressive relapse. Behav Res Ther 2008;46:1142-1150.

Lewinsohn PM, Allen NB, Seeley JR, Gotlib IH: First onset versus recurrence of depression: differential processes of psychosocial risk. J Abnorm Psychol 1999;108:483489.

Miranda J, Gross JJ, Persons JB, Hahn J: Mood matters: negative mood induction activates dysfunctional attitudes in women vulnerable to depression. Cognit Ther Res 1998;22:363-376.

Miranda J, Persons JB: Dysfunctional attitudes are moodstate dependent. J Abnorm Psychol 1988:97:76-79.

Missel P, Sommer G: Depression and self-verbalization. Cognit Ther Res 1983;7:141-148.

Nolen-Hoeksema S, Girgus JS, Seligman ME: Predictors and consequences of childhood depressive symptoms: a 5-year longitudinal study. J Abnorm Psychol 1992; 101:405-422.
Oei TPS, Shuttlewood GJ: Specific and non-specific factors in psychotherapy: a case of cognitive therapy for depression. Clin Psychol Rev 1996;16:83-103.

Oei TPS, Sullivan L: Cognitive changes following recovery from depression in a group cognitive-behaviour therapy program. Aust N Z J Psychiatry 1999;33:407-415.

Pössel P, Seemann S, Hautzinger M: Evaluation eines deutschsprachigen Instruments zur Erfassung positiver und negativer automatischer Gedanken. Z Klin Psychol Psychother 2005;24:27-34.

Pössel P, Knopf K: An experimental test of the maintenance and vulnerability hypothesis of depression in consideration of the cognitive hierarchy. Depress Anxiety 2008;25:47-55.

Rojas R, Geissner E, Schirm E, Hautzinger M: Kognitive Reaktivität und Stressbelastung als Prädiktoren eines Rezidivs bei remittiert depressiven Personen. Z Klin Psychol Psychother 2014;43:17-26.

Rush AJ, Trivedi MH, Ibrahim HM, et al: The 16-Item Quick Inventory of Depressive Symptomatology (QIDS), clinician rating (QIDS-C), and self-report (QIDS-SR): a psychometric evaluation in patients with chronic major depression. Biol Psychiatry 2003; 54: 573-583.

Schauenburg H, Clarkin J: Rückfälle bei depressiven Erkrankungen - Sind psychotherapeutische "Erhaltungsstrategien» sinnvoll? Z Psychosom Med Psychother 2003;49:377-390.

Scher CD, Ingram RE, Segal ZV: Cognitive reactivity and vulnerability: empirical evaluation of construct activation and cognitive diatheses in unipolar depression. Clin Psychol Rev 2005;25:487-510.

Segal ZV, Gemar M, Williams S: Differential cognitive response to a mood challenge following successful cognitive therapy or pharmacotherapy for unipolar depression. J Abnorm Psychol 1999;108:3-10.

Segal ZV, Kennedy S, Gemar M, et al: Cognitive reactivity to sad mood provocation and the prediction of depressive relapse. Arch Gen Psychiatry 2006;63:749-755.

Shiraishi S: Cognitive therapy for reducing and preventing depressive moods: a practical study with undergraduates. Jpn J Educ Psychol 2005;53:252-262.

Szentagotai A, Freeman A: An analysis of the relationship between irrational beliefs and automatic thought in predicting distress. J Cogn Behav Psychother 2007;7: $1-9$.

Thomsen DK: The association between rumination and negative affect: a review. Cogn Emot 2006;20:12161235

Vernon PA: Der Zahlen-Verbindungs-Test and other Trail-Making correlates of general intelligence. Pers Individ Dif 1993;14:35-40.

Winkeljohn Black S, Pössel P: Integrating Beck's cognitive model and the response style theory in an adolescent sample. J Youth Adolesc 2015;44:195-210.

Wittchen H-U, Zaudig M, Fydrich T: Strukturiertes Klinisches Interview für DSM-IV: Achse I und Achse II. Göttingen, Hogrefe, 1997.

Zettle RD, Rains JC, Hayes SC: Processes of change in acceptance and commitment therapy and cognitive therapy for depression: a mediation reanalysis of Zettle and Rains. Behav Modif 2011:35:265-283. 